\title{
LED-induced fluorescence diagnostics for turbine and combustion engine thermometry
}

\author{
S. W. Allison,* D. L. Beshears, ** M. R. Cates, M. Paranthaman \\ Engineering Technology Division \\ Oak Ridge National Laboratory \\ Knoxville, TN. 37932 \\ G. T. Gillies \\ Dept. of Physics \\ The University of Virginia \\ Charlottesville, VA. 22901
}

\begin{abstract}
Fluorescence from phosphor coatings is the basis of an established technique for measuring temperature in a wide variety of turbine and combustion engine applications. Example surfaces include blades, vanes, combustors, intake valves, pistons, and rotors. Many situations that are remote and noncontact require the high intensity of a laser to illuminate the phosphor, especially if the surface is moving. Thermometric resolutions of $0.1 \mathrm{C}$ are obtainable, and some laboratory versions of these systems have been calibrated against NIST standards to even higher precision. To improve the measurement signal-to-noise ratio, synchronous detection timing has been used to repeatedly interrogate the same blade in a high speed rotating turbine. High spatial resolution can be obtained by tightly focusing the interrogation beam in measurements of static surfaces, and by precise differential timing of the laser pulses on rotating surfaces. We report here the use of blue light emitting diodes (LEDs) as an illumination source for producing useable fluorescence from phosphors for temperature measurements. An LED can excite most of the same phosphors used to cover the temperature range from 8 to $1400 \mathrm{C}$. The advantages of using LEDs are obvious in terms of size, power requirements, space requirements and cost. There can also be advantages associated with very long operating lifetimes, wide range of available colors, and their broader emission bandwidths as compared to laser diodes. Temperature may be inferred either from phase or time-decay determinations.
\end{abstract}

Keywords: thermographic phosphor, temperature, light emitting diode, LED, fluorescence

\subsection{INTRODUCTION}

Phosphor thermometry exploits the thermal dependence of the characteristic fluorescence of materials. Among the properties that are temperature dependent are spectral distributions, intensities, line-widths, line positions, and decay rates. It is usually the case that fluorescence decay time, $\tau$, also known as lifetime, is the parameter that is measured to determine the temperature, $\mathrm{T}$. Under pulsed excitation, the time dependence of fluorescence amplitude, I, is usually a single exponential:

$$
I=I_{0} \exp \left(\frac{-t}{\tau}\right) \text { where } \tau=f(T)
$$

Gratten and Zhang ${ }^{1}$ have authored a monograph which is a survey of the method with particular emphasis on the use of fluorescence sensing of temperature with fiber optics. A review article by Allison and Gillies ${ }^{2}$ surveys the field with special attention paid to noncontact applications. Any fluorescent material will exhibit atemperature dependence in some range, and thus, in addition to phosphor powders; liquids, glasses and crystals have all been used for thermometry purposes. Phosphor powders are easily mixed with binder materials for coating the surface of interest. Or, they may in some instances be direct-

*allisonsw@ ornl.gov; 1865 946-1287 fax 1865 946-1292; http://www.ornl.gov/phosphors; Oak Ridge National Laboratory, 2360 Cherahala Blvd.; MS-6472 CVO; Knoxville, TN., USA; 37932;

** beshearsdl@ornl.gov; 1865 946-1288 same fax and address 
deposited via electron-beam or ion-beam sputtering. A typical system will consist of an excitation light source, an optical system for delivering the excitation to the surface of interest, an optical means for directing the resultant fluorescence to a detector, and a data analysis system.

This paper contains a discussion with examples of issues and considerations with regard to utilizing blue LEDs as light sources for fluorescence-based thermometry of turbine and combustion engines. The last section discusses phase modulation techniques that are enabled with continuous emitting light emitting diodes.

The first consideration is the expected temperature range. Phosphors that are made from refractory materials such as garnets and oxides are usually best for temperatures above about $700 \mathrm{C}$. A wide variety of other luminescent materials may be used for the lower ranges. Choice of phosphor may further be delimited by the second consideration, whether or not the surface of interest is moving. For surfaces moving at high speeds, a fluorescing area may move into and out of the field-of-view of the collection optics. This is especially crucial for imaging applications of moving surfaces. In such cases, motion of a few tens of microns may blur the image. The received signal will exhibit a time dependence governed by this motion in addition to the exponential time dependence. For a phosphor with a sufficiently short decay time, this is not a problem.

Non-contact applications of phosphor thermometry formerly required laser sources. However, recent tests with a blue LED peaked near $450 \mathrm{~nm}$ showed that it is possible to excite a wide variety of these materials with simple setups such as the one below in Figure 1. There, an LED pumps a $1 \mathrm{~mm}$ diameter optical fiber which illuminates a sintered $\mathrm{Y}_{2} \mathrm{O}_{3}:$ Eu sample seen in the foreground. In the upper left hand corner, a photomultiplier, with bandpass filter, detects fluorescence. The LED is a gallium nitride type from Ledtronics, part number BP280CWB1K-3.6Vf-050T, rated at 1000 mcd luminous intensity. All efforts in this lab involve inorganic phosphors which consist of a host material and an activator atom, usually either a rareearth or transition metal. Excitable phosphor hosts include yttrium garnets, yttrium oxide, oxysulfides, vanadates, and yttrium phosphates with activators of $\mathrm{Eu}, \mathrm{Tb}, \mathrm{Dy}, \mathrm{Pr}, \mathrm{Sm}$, and $\mathrm{Ce}$. To date the only materials not excitable by a blue LED have been phosphors with emission bands below $450 \mathrm{~nm}$ and absorption well below $380 \mathrm{~nm}$.

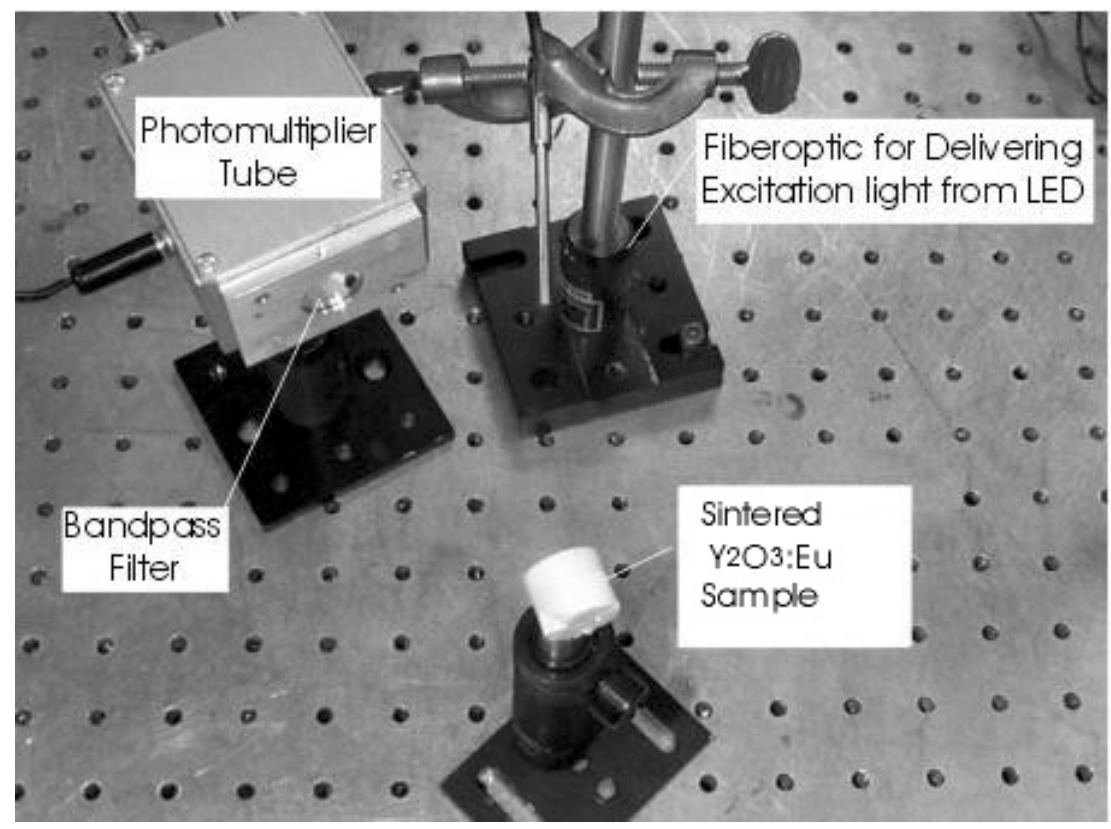

Figure 1. Sintered $\mathrm{Y}_{2} \mathrm{O}_{3}$ :Eu sample illuminated by LED-pumped fiberoptic.

\subsection{Long Decay Time}

A very important host matrix material is $\mathrm{Y}_{3} \mathrm{Al}_{5} \mathrm{O}_{12}$, commonly known by the acronym YAG which stands for yttrium aluminum garnet. The phosphor YAG:Cr is a prime example of a material that luminesces efficiently when excited by a blue LED. Figure 2 shows the excitation spectrum of YAG:Cr in relation to the emission spectrum of the blue LED used in the tests described here. The sample was prepared in our organization by Matsubara. ${ }^{3}$ The LED emission peak falls between the 
two absorption maxima of the YAG:Cr. Nonetheless, sufficient overlap exists to yield bright emission in the characteristic band of this material which is centered at $707 \mathrm{~nm}$. At room temperature, the decay time is about $4 \mathrm{~ms}$. A surface moving at $1 \mathrm{~m} / \mathrm{s}$ would move about $4 \mathrm{~mm}$ during one decay constant. This material exhibits temperature dependence over a wide range and the decay times are somewhat shorter as depicted in Figure 3. It shows several fluorescence signals at the high end of its operable range to $680 \mathrm{C}$ where the decay time is about $3 \mu \mathrm{s}$. The LED pulse depicted is about $10 \mu \mathrm{s}$ duration. It is seen that the fluorescence signal builds in time as energy is absorbed from the LED. The test arrangement used a single LED about 3 $\mathrm{cm}$ from a coated heating element. A $5 \mathrm{~cm}$ focal length lens images the fluorescence onto a photomultiplier tube with an intervening filter passing all fluorescence wavelengths greater than $665 \mathrm{~nm}$.

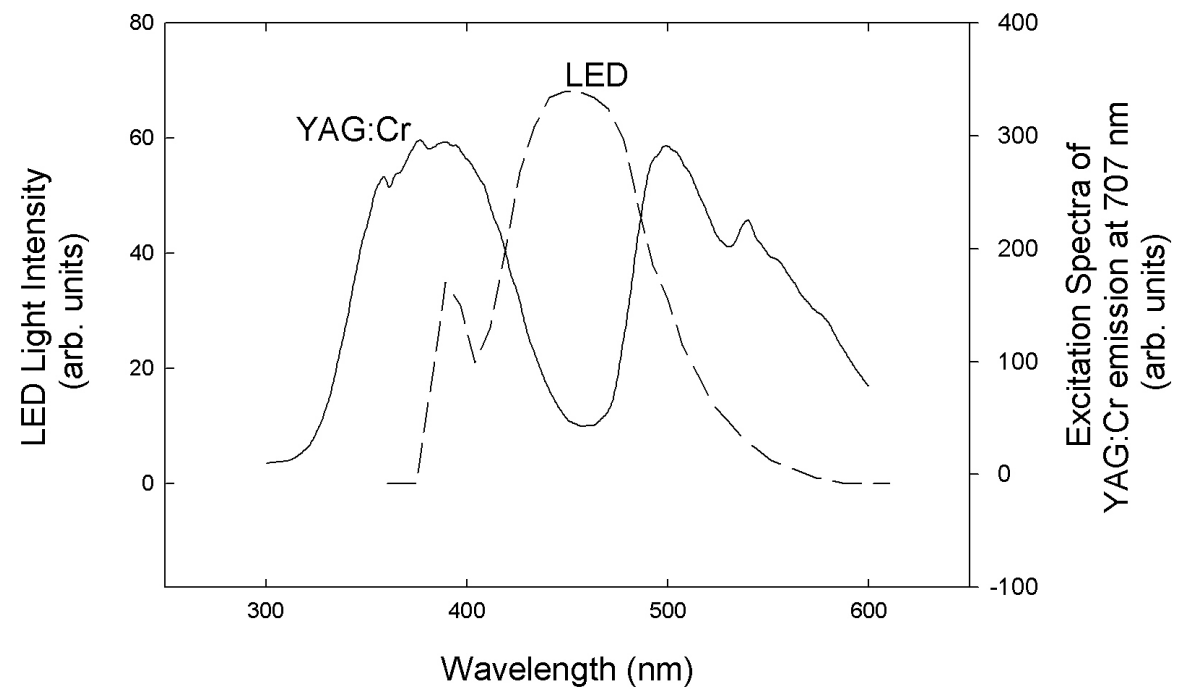

Figure 2. YAG:Cr excitation spectrum and LED emission spectrum.

It should be noted that LED excitation is particularly suited to the longer decay time phosphors since there is a long build up time for the emission. Thus, it would require a 3 ns laser pulse to be about 3000 times brighter than the LED in order to produce fluorescence of the same amplitude as in figure 3.

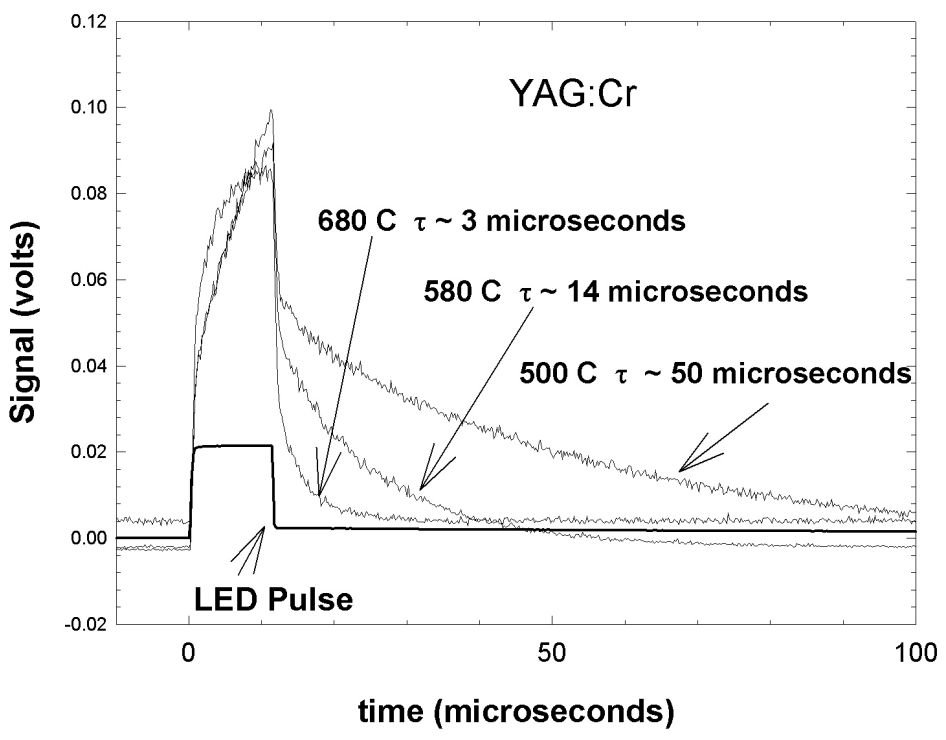

Figure 3. YAG:Cr emission time dependence with blue LED pulse. 


\subsection{SHORT DECAY TIME}

For transient measurements and for surfaces that are moving rapidly, short decay time luminescence is desirable. Cerium doped phosphors exhibit the shortest decay times of commercially viable phosphor materials ${ }^{4}$. A number of garnet hosts have been tested in our lab with Ce as an activator. Decay times rang from a few ns to about $60 \mathrm{~ns}$. The absorption bands are typically very broad and in the blue, with the fluorescence likewise broadband and appearing green or blue-green to human vision. As a class, with some exceptions, they are efficiently excited by a blue LED. Figure 4 shows fluorescence decay from one of these, a YAG:Ce formulation with the aluminum replaced in the ratio shown in the figure by gallium, Ga. The use of gallium has the effect of tuning the range of the temperature dependence. The emission decay time is $60 \mathrm{~ns}$ at about $-40 \mathrm{C}$ and decreases to about $10 \mathrm{~ns}$ at ambient $(23 \mathrm{C})$, the limit of the time response of the oscilloscope used for the testing.

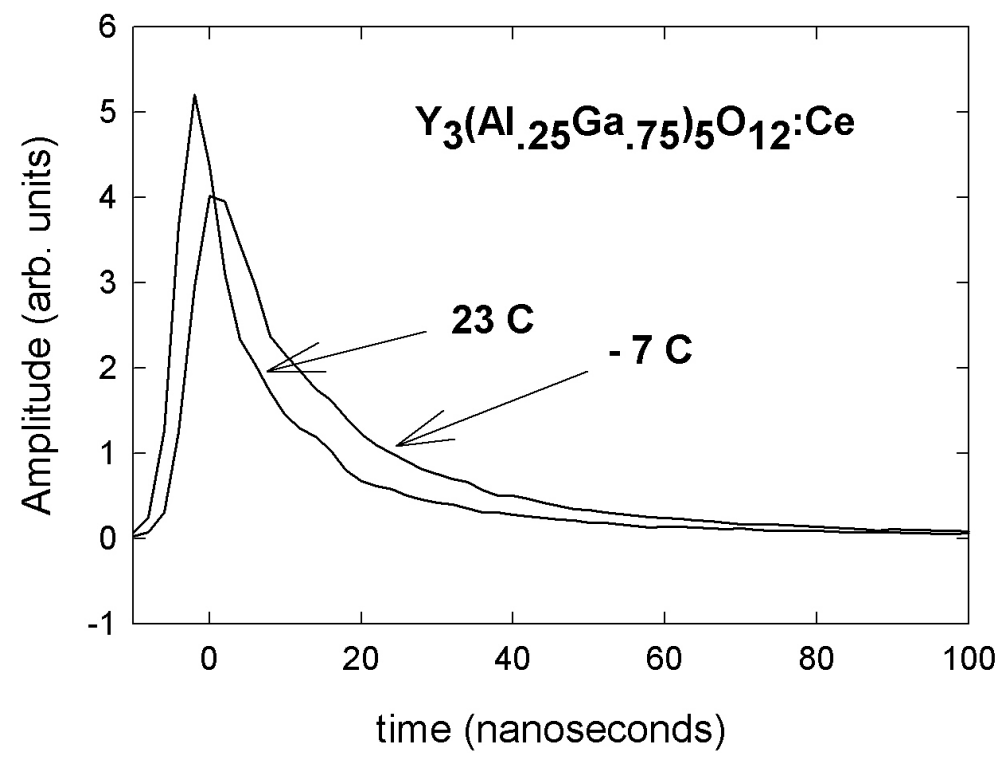

Figure 4. Short decay time phosphor with high gallium content.

\subsection{TEMPERATURE DEPENDENCE COMPARISONS}

Figure 5 shows the temperature vs decay time (calibration curve) for several selected phosphors that are excitable by a blue LED. Data points are shown as well as linear regression fits to the data, shown as the solid line. The dotted line is the projected temperature dependence at higher temperatures. For stationary applications, the YAG:Cr can cover a wide temperature range. Also plotted here is a representative $\operatorname{Pr}$ activated phosphor, $\mathrm{Y}_{2} \mathrm{O}_{2} \mathrm{~S}$ :Pr. With the exception of Ce materials, Pr phosphors exhibit the shortest emission decays of rare earths. Unquenched decay times range from about 3 to 8 $\mu \mathrm{s}$. The two different YAG:Ce phosphors in the figure illustrate how the temperature dependence can be controlled by adjusting the gallium content. If there is no gallium, YAG:Ce does not show temperature dependence until nearly $200 \mathrm{C}$.

Previous work in car engines has involved such surfaces as the crown of a piston, an intake valve, and inside a catalytic converter. ${ }^{5,6}$ The top of the figure indicates the temperature ranges of the various surfaces. This provides a means to aid in choosing a phosphor material for a given surface and temperature range and desired decay time. Turbine engine and certain aerospace applications involve much higher temperatures than automobile applications, usually in excess of $600 \mathrm{C}$. Quite a few other phosphor materials could have been depicted in the figure. The ones chosen were selected in order to show the range of decay times available and the general temperature range achievable with LED excitation by our group to date. Above about $700 \mathrm{C}$, decay time measurements with low brightness LEDs are rendered more difficult by blackbody radiation since the latter may exceed by several orders of magnitude the fluorescence signal. However, phase-based methods to be described below may be more appropriate for such higher temperatures. 


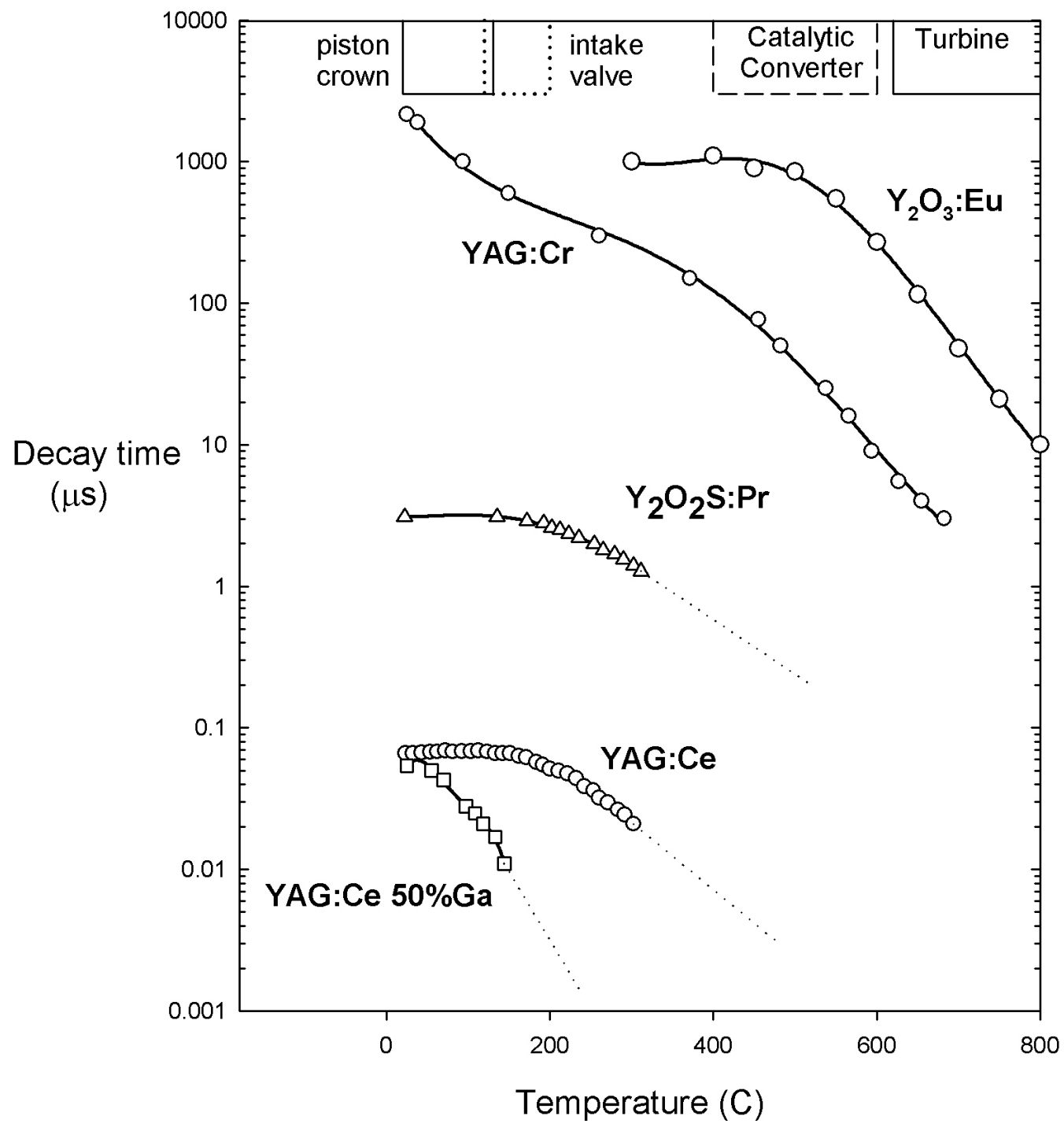

Figure 5. Temperature dependence of selected phosphors and decay time comparisons.

\subsection{PHASE-BASED METHODS}

A well-known technique for determining decay time that is already commercially available in instruments like spectrophotometers uses the fluorescent phase shift from a modulated interrogating source. For an excitation source sinusoidally modulated at frequency $\mathrm{f}$, this phase shift, $\phi$, is mathematically correlated with the fluorescence decay and from it temperature can be inferred by the relation:

$$
\tan (\phi)=2 \cdot \pi \cdot \mathrm{f} \cdot \tau
$$

This is illustrated in Figure 6. In some applications the phase shift approach may be ideal, but in others, especially where the measurement environment is far more inhospitable than a laboratory, new techniques may be called for. The following are 
related measurement approaches. (Reference 1, chapter 3, contains an analysis of some of these and other phase and pulse modulation methods.)

\section{Sinusoidal Excitation}

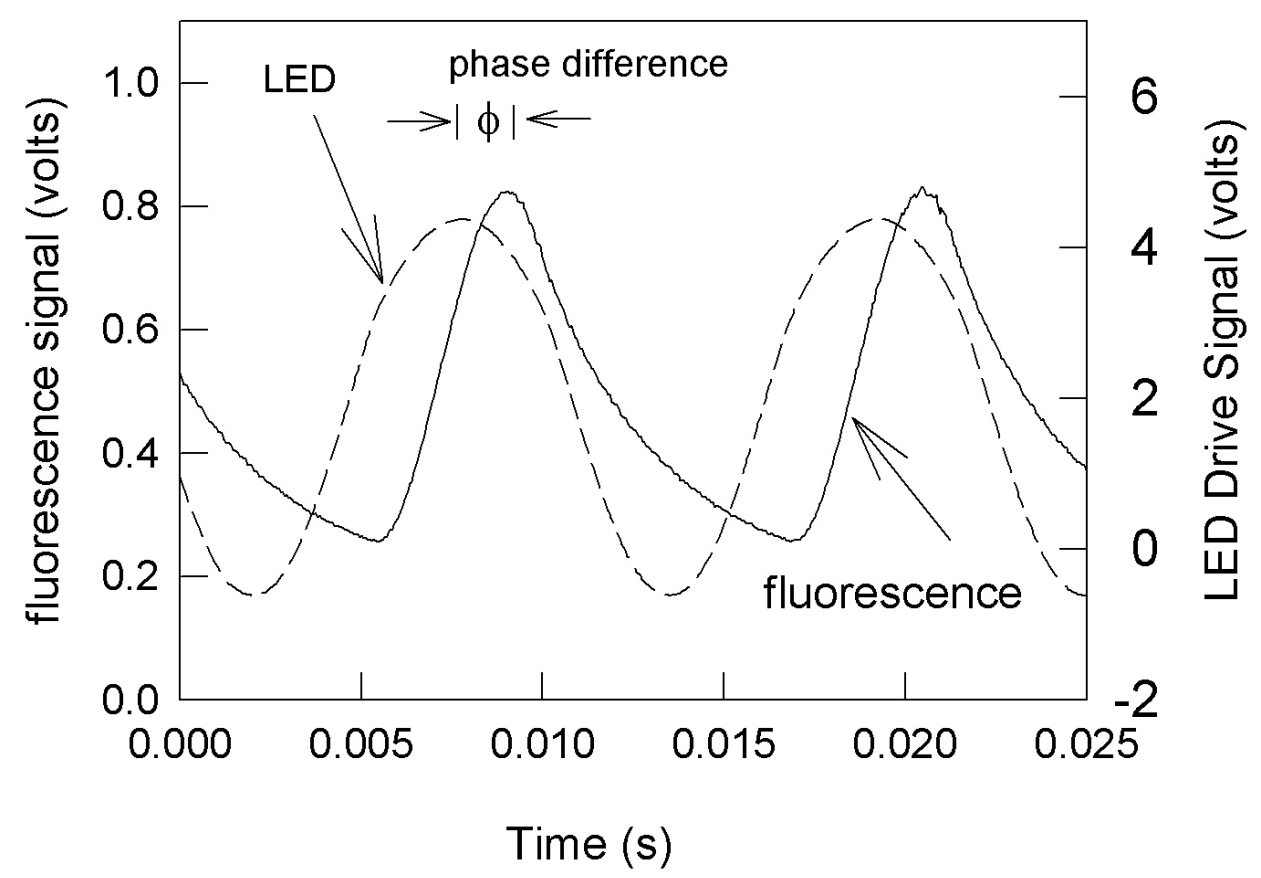

Figure 6. Time dependence of fluorescence from $\mathrm{Mg}_{4} \mathrm{FGeO}_{6}: \mathrm{Mn}$ excited sinusoidally by LED.

5.1 Square wave modulation and ratio of "peak to valley". When the square wave train that stimulates fluorescence is comprised of light-on and light-off periods that are on the order of fluorescent lifetimes the fluorescence signal will not return to its base line during light-off periods. An example is seen in Figure 6. The peak to valley or maximum to minimum is a function of fluorescence lifetime which in turn is correlated with temperature. Consequently, if the square wave train light-on to light-off voltage or power ratio is used as a reference, the maximum light to minimum light in the train of fluorescence emissions correlates to temperature and can be calibrated correspondingly.

Another variant on the technique is to establish a desired peak to valley ratio for the fluorescence, adjusting the stimulating waveform frequency until it is obtained, the waveform frequency then being correlated to temperature.

5.2 Generalized modulation waveform and phase shift analysis. When an arbitrary stimulating waveform is used for the LED power source, it is possible to cast the waveform in Fourier space by performing the transform into phase space, which consists of an infinite series of orthogonal sine waves with coefficients weighted by the particular shape. Similarly, the resultant fluorescent waveform can be Fourier analyzed. The phase shift between each Fourier component of the stimulating waveform and its counterpart in the fluorescence waveform can be determined. Those phase shifts are correlated to fluorescence lifetime, consequently, the temperature.

The simplest case of the generalized modulation waveform is a constant amplitude sine wave (with the single Fourier component). It is this simple case that is approximated in commercial photometric analyzers using phase shifts. However, there could easily be circumstances where a series of narrow, high-intensity pulses may be a far better interrogation method in actual applications. Similarly, some other waveforms (e.g., sawtooths, rectified sine waves, etc.) may be either optimum for the particular fluorescent response that must be evoked or may be the best choice for the particular power source, noise environment, etc. By doing the full analysis any repeated waveform can be used to extract temperature. 
5.3 Motion Modulation. Motion can be used to advantage in this scheme. As a luminescing surface moves from the point where it is illuminated, the emission amplitude decreases. The difference in amplitude of two separated detectors is related to the time for the illuminated area to move from one detector to the other. Hence, the decaying waveform is sampled at two different times. This can be correlated with the characteristic fluorescence decay time. Visually, for a high-speed surface, the fluorescence appears as a stripe. An interesting example of this is provided by $\mathrm{La}_{2} \mathrm{O}_{2} \mathrm{~S}: \mathrm{Eu}$, a phosphor for which different emission lines and colors have different decay times. When coated onto a disk moving a sufficient speed, a series of color bands are seen with the more blue lines visible close to the illumination region and the redder, longer lived lines further away.

5.4 Interrogation color ratio. Here two (or more) light wavelength bands are used for the LEDs (or similar sources) stimulating the fluorescence. Because the excitation spectra for many phosphors are temperature dependent the resultant amplitudes of fluorescence induced by two separate wavelength bands will in general be functions of temperature. The calibration is also parameterized by the interrogation wavelength bands and waveform characteristics.

The measurement technique in this embodiment could either record emission ratios or record intensity ratios of the stimulating wavelengths required to meet some fluorescence condition, such as producing a prescribed emission intensity ratio, matching intensities of two lines on a readout, or another equivalent result.

5.5 Fluorescence ratio from sequential time-on waveform variation. Here the train of (for simplicity) square waves has at least two different time-on periods, so that the fluorescences stimulated by each of the two different periods are different in characteristic from the other. The peak to valley, fluorescence decay, or the phase shift could be measured for each, then the two compared. The results are functions of temperature and can be calibrated in advance. The calibrations have parameters which include, LED color, both time-on periods, the time-off periods, and other means.

\subsection{CONCLUSIONS}

Phosphor thermometry offers the measurement specialist a noncontact, remote means for interrogating surface temperatures. The method is capable of achieving high-resolution thermal mappings with instrumentation that can be straightforwardly calibrated and traced to NIST standards. The advent of blue LEDs as excitation sources has done much to improve the versatility of this approach over previous versions of such systems that relied on pulsed nitrogen/ultraviolet lasers to illuminate the phosphor target. We have explored the application of several classes of phosphors to measurement scenarios compatible with turbine and combustion engine testing environments, and we have presented representative data that show how the fluorescence amplitudes and decay times of selected phosphors depend on temperature. This work is part of our overall efforts aimed at developing novel uses of these materials in a variety of situations where robust measurement systems are needed.

\subsection{ACKNOWLEDGEMENTS}

The Oak Ridge National Laboratory is managed by UT-Battelle, LLC for the U.S. Department of Energy Under contract DE-AC05-00OR22725.

\subsection{REFERENCES}

\footnotetext{
${ }^{1 .}$ K. T. V. Grattan and Z. Y. Zhang, Fiber Optic Fluorescence Thermometry, Chapman and Hall, London, UK, 1995.

2. S. W. Allison and G. T. Gillies, "Remote thermometry with thermographic phosphors: Instrumentation and applications." Rev. Sci. Instrum, Vol. 68(7), pp.2615-2650, 1997.

${ }^{3}$ I. Matsubara, M. Paranthaman, S. W. Allison, M. R. Cates, D. L. Beshears, D. E. Holcomb, Cr-doped $Y_{3} \mathrm{Al}_{5} \mathrm{O}_{12}$ phosphors by heterogeneous precipitation methods and their luminescent properties, Materials Research Bulletin 35(2000)217-224.

${ }^{4}$ Shionoya S and Yen W M eds Phosphor Handbook 1999 CRC Press 189, p.496.
} 
${ }^{5}$ Armfield, J. S., Beshears, D. L., "Phosphor Thermometry for Internal Combustion Engines", Society of Automotive Engineers Technical Paper No. 971642, Presented at the 1997 Society of Automotive Engineers Spring Fuel and Lubricants Meeting, Dearborn MI, May 5-7, 1997.

${ }^{6}$ Armfield, J. F., Domingo, N., Storey, J. M., Allison, S. W., Beshears, D. L., Cates, M. R., "Powertrain Component Temperature Measurement via Phosphor Themometry", Presented at the World Car Conference, January 19-22, 1997, Riverside California, Ref. No. 97WCC018. 